\title{
Parameter-free Structural Diversity Search
}

\author{
JINBIN HUANG, Hong Kong Baptist University, China \\ XIN HUANG, Hong Kong Baptist University, China \\ YUANYUAN ZHU, Wuhan University, China \\ JIANLIANG XU, Hong Kong Baptist University, China
}

\begin{abstract}
The problem of structural diversity search is to find the top- $k$ vertices with the largest structural diversity in a graph. However, when identifying distinct social contexts, existing structural diversity models (e.g., $t$-sized component, $t$-core, and $t$-brace) are sensitive to an input parameter of $t$. To address this drawback, we propose a parameter-free structural diversity model. Specifically, we propose a novel notation of discriminative core, which automatically models various kinds of social contexts without parameter $t$. Leveraging on discriminative cores and $h$-index, the structural diversity score for a vertex is calculated. We study the problem of parameterfree structural diversity search in this paper. An efficient top- $k$ search algorithm with a well-designed upper bound for pruning is proposed. Extensive experiment results demonstrate the parameter sensitivity of existing $t$-core based model and verify the superiority of our methods.
\end{abstract}

\section{ACM Reference Format:}

Jinbin Huang, Xin Huang, Yuanyuan Zhu, and Jianliang Xu. 2019. Parameter-free Structural Diversity Search. 1, 1 (October 2019), 13 pages. https://doi.org/10.1145/nnnnnnn.nnnnnnn

\section{INTRODUCTION}

Nowadays, information spreads quickly and widely on social networks (e.g., Twitter, Facebook). Individuals are usually influenced easily by the information received from their social neighborhoods [14]. Recent studies show that social decisions made by individuals often depend on the multiplicity of social contexts inside his/her contact neighborhood, which is termed as structural diversity [25]. Individuals with larger structural diversity, are shown to have higher probability to be affected in the process of social contagion [25]. Structural diversity search, finding the individuals with the highest structural diversity in graphs, has many applications such as political campaigns [15], viral marketing [17], promotion of health practices [25], facebook user invitations [25], and so on.

In the literature, several structural diversity models (e.g., $t$-sized component, $t$-core and $t$-brace) need an input of specific parameter $t$ to model distinct social contexts. A social context is formed by a number of connected users. The component-based structural diversity [25] regards each connected component whose size is larger than $t$ as a social context. Another core-based structural diversity model is defined based on $t$-core. A $t$-core is the largest subgraph such that each vertex has at least $t$ neighbors within $t$-core. The core-based structural diversity model regards each maximal connected $t$-core as a distinct social context. Fig. 1 shows the contact neighborhood (ego-network)

Authors' addresses: Jinbin Huang, Hong Kong Baptist University, Hong Kong, China, jbhuang@comp.hkbu.edu.hk; Xin Huang, Hong Kong Baptist University, Hong Kong, China, xinhuang@comp.hkbu.edu.hk; Yuanyuan Zhu, Wuhan University, Wuhan, China, yyzhu@whu.edu.cn; Jianliang Xu, Hong Kong Baptist University, Hong Kong, China, xujl@comp.hkbu.edu. hk.

Permission to make digital or hard copies of all or part of this work for personal or classroom use is granted without fee provided that copies are not made or distributed for profit or commercial advantage and that copies bear this notice and the full citation on the first page. Copyrights for components of this work owned by others than ACM must be honored Abstracting with credit is permitted. To copy otherwise, or republish, to post on servers or to redistribute to lists, requires prior specific permission and/or a fee. Request permissions from permissions@acm.org.

(c) 2019 Association for Computing Machinery.

XXXX-XXXX/2019/10-ART \$15.00

https://doi.org/10.1145/nnnnnnn.nnnnnnn

, Vol. 1, No. 1, Article . Publication date: October 2019. 


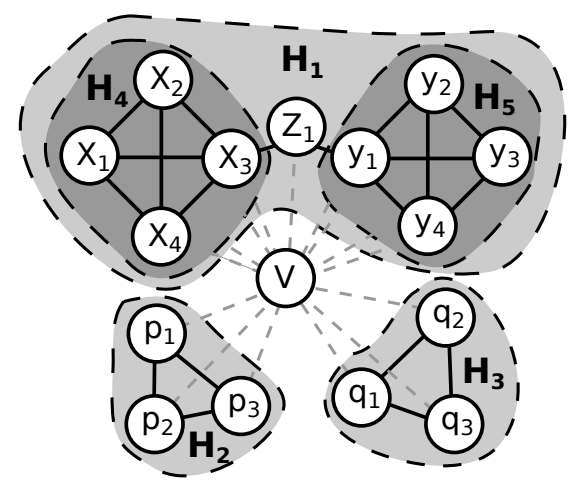

Fig. 1. The ego-network $G_{N(v)}$ of vertex $v$

$G_{N(v)}$ of a user $v$. All vertices and edges in ego-network $G_{N(v)}$ are in solid lines. Consider the core-based structural diversity model and parameter $t=2$. Subgraphs $H_{1}, H_{2}$ and $H_{3}$ are maximal connected 2-cores. $H_{1}, H_{2}$, and $H_{3}$ are regarded as 3 distinct social contexts. Thus, the core-based structural diversity of $v$ is 3 .

This paper proposes a new parameter-free structural diversity model based on the core-based model [12] and h-index measure [11]. Our parameter-free model does not need the input of parameter $t$ any more. This avoids suffering from the limitations of setting parameter $t$. We show two major drawbacks of the $t$-core based model as follows.

- Sensitivity of $\mathbf{t}$-core based model. The number of social contexts is sensitive to parameter $t$. On the one hand, if $t$ is set to a large value, it may discard small and weakly-connected social contexts; On the other hand, if $t$ is set to a small value, it may have weak ability of recognizing strongly-connected social contexts fully. Consider the contact neighborhood $G_{N(v)}$ of a user $v$ in Fig. 1 . When $t=2$, the structural diversity of $v$ is 3 . When $t=3, H_{2}$ and $\mathrm{H}_{3}$ are 2-cores and disqualified for social contexts, due to the requirement of social contexts as 3-core. Meanwhile, $H_{1}$ is decomposed as two components of 3-core as $H_{4}$ and $H_{5}$. Thus, the structural diversity of $v$ becomes 2 . However, when $t \geq 4$, the structural diversity of $v$ is 0 . This example clearly shows the sensitivity of structural diversity w.r.t. parameter $t$.

- Inflexibility of t-core based model. Structural diversity model lacks flexibility for different vertices using the same parameter $t$. Generally, different social contexts should not be modeled and quantified using the same criteria of parameter $t$. For example, in a social network, the social contexts of a famous singer and a junior student can be dramatically different in terms of size and density. Thus, it is difficult to choose one consistent value $t$ for different vertices in a graph. In Fig. $1, H_{1}$ can be decomposed into two social contexts $H_{4}$ and $H_{5}$, which requires the setting of $t=3$. However, the identification of $H_{2}$ and $H_{3}$ requires $t=2$. This indicates the necessary of personalized parameter $t$ for different social contexts.

To address the above two limitations, we define a novel notation of discriminative core to represent each distinct social context without inputing any parameters. Specifically, a discriminative core is a densest and maximal connected subgraph inside a user's contact neighborhood. It can be regarded as a criteria for representing unique and strong social context. However, the distribution of discriminative cores in two users' contact neighborhoods can be totally different in terms of density and quantity, which cannot be compared directly. To tackle this issue, we propose a new structural diversity model based on $h$-index. In the literature, the $h$-index is defined as the maximum number of $h$ such that a researcher has published $h$ papers whose citations have at least $h$ [11]. We apply the similar idea to measure structural diversity in ego-networks. Given a vertex $v$, the structural diversity of $v$ is the largest number $h$ such that there exists at least $h$ discriminative cores with 
coreness at least $h$. In this paper, we study the problem of top- $k h$-index based structural diversity search, which finds $k$ vertices with largest $h$-index based structural diversity. To summarize, we make the following contributions:

- We propose a novel definition of discriminative core to provide a parameter-free scheme for identifying social contexts. To simultaneously measure the quantity and strength of social contexts in one's contact neighborhood, we propose a new $h$-index based structural diversity model. We formulate the problem of top- $k h$-index based structural diversity search in a graph. (Section 3)

- We propose a useful approach for computing the $h$-index based structural diversity score $h(v)$ for a vertex $v$ and give a baseline algorithm for solving the top- $k$ structural diversity search problem. (Section 4)

- Based on the analysis of the discriminative core structure and the property of $h$-index, we design an upper bound of $h(v)$. Equipped with the upper bound, we propose an efficient top- $k$ search framework to improve the efficiency. (Section 5).

- We conduct extensive experiments on four real-world large datasets to demonstrate the parameter sensitivity of the existing core-based structural diversity model and verify the effectiveness of our proposed model. Experiment results also validate the efficiency of our proposed algorithms. (Section 6)

\section{RELATED WORK}

This work is related to the studies of structural diversity search and $k$-core mining.

Structural Diversity Search. In [25], Ugander et al. studied the structural diversity models in the real-world applications of social contagion. The problem of top- $k$ structural diversity search is proposed and studied by Huang et al. $[12,13]$. The goal of the problem is to find $k$ vertices with the highest structural diversity scores. Two structural diversity models based on $t$-sized component and $t$-core respectively are studied w.r.t. a parameter threshold $t$. Recently, Chang et al. [4] proposed fast algorithms to address structural diversity search by improving the efficiency and scalability of the methods [13]. Cheng et al. [5] propose an approach of diversity-based keyword search to solve the mashup construction problem. Different from above studies, we propose a parameter-free structural diversity model based on the novel definition of discriminative cores, which avoids suffering from the difficulties of parameter tuning.

K-Core Mining. There exist lots of studies on $k$-core mining in the literature. $k$-core is a definition of cohesive subgraph, in which each vertex has degree at least $k$. The task of core decomposition is finding all non-empty $k$-cores for all possible $k$ 's. Batagelj et al. [2] proposed an in-memory algorithm of core decomposition. Core decomposition has also been widely studied in different computing environment such as external-memory algorithms [6], streaming algorithms [23], distributed algorithms [22], and I/O efficient algorithms [26]. The study of core decomposition is also extended to different types of graphs such as dynamic graphs [1,16], uncertain graphs [3], directed graphs [19], temporal graphs [27], and multi-layer networks [9]. Recently, core maintenance in dynamic graphs has attracted significant interest in the literature $[1,20,28]$.

\section{PROBLEM STATEMENT}

In this section, we formulate the problem of $h$-index based structural diversity search.

\subsection{Preliminaries}

We consider an undirected and unweighted simple graph $G=(V, E)$, where $V$ is the set of vertices and $E$ is the set of edges. We denote $n=|V|$ and $m=|E|$ as the number of vertices and edges in 
$G$ respectively. W.l.o.g. we assume the input graph $G$ is a connected graph, which implies that $m \geq n-1$. For a given vertex $v$ in a subgraph $H$ of $G$, we define $N_{H}(v)=\{u$ in $H:(u, v) \in E(H)\}$ as the set of neighbors of $v$ in $H$, and $d_{H}(v)=\left|N_{H}(v)\right|$ as the degree of $v$ in $H$. We drop the subscript of $N_{G}(v)$ and $d_{G}(v)$ if the context is exactly $G$ itself, i.e. $N(v), d(v)$. The maximum degree of graph $G$ is denoted by $d_{\text {max }}=\max _{v \in V} d_{G}(v)$.

Given a subset of vertices $S \subseteq V$, the subgraph of $G$ induced by $S$ is denoted by $G_{S}=(S, E(S))$, where the edge set $E(S)=\{(u, v) \in E: u, v \in S\}$. Based on the definition of induced subgraph, we define the ego-network $[8,21]$ as follows.

Definition 3.1. (Ego-network) Given a vertex $v$ in graph $G$, the ego-network of $v$ is the induced subgraph of $G$ by its neighbors $N(v)$, denoted by $G_{N(v)}$.

In the literature, the term "neighborhood induced subgraph" [12] is also used to describe the ego-network of a vertex. For example, consider the graph $G$ in Fig. 1. The ego-network of vertex $v$ is shown in the gray area of Fig. 1, which excludes $v$ itself with its incident edges. The $t$-core of a graph $G$ is the largest subgraph of $G$ in which all the vertices have degree at least $t$. However, the $t$-core of a graph can be disconnected, which may not be suitable to directly depict social contexts. Hence, we define the connected $t$-core as follows.

Definition 3.2. (Connected $t$-Core) Given a graph $G$ and a positive integer $t$, a subgraph $H \subseteq G$ is called a connected $t$-Core iff $H$ is connected and each vertex $v \in V(H)$ has degree at least $t$ in $H$.

Given a parameter $t$, the core-based structural diversity model treats each maximal connected $t$-core as a distinct social context [12][25]. To measure the structural diversity of an ego-network, one essential step is to tune a proper value for parameter $t$. However, such parameter setting is not easy and even critically challenging. The following example illustrates it.

Example 3.3. Fig. 1 shows an ego-network $G_{N(v)}$ of vertex $v$. Given an integer $t=2$, three maximal connected 2-core $\left(H_{1}, H_{2}\right.$ and $\left.H_{3}\right)$ will be treated as distinct social contexts. The core-based structural diversity of $v$ is 3 . When we set $t=3$, the core-based structural diversity of $v$ will be 2 , since $H_{4}$ and $H_{5}$ will be treated as two distinct social contexts. In this case, $H_{2}$ and $H_{3}$ are no longer treated as social contexts. If we set $t$ to be some values higher than 3 , no social contexts can be identified. The core-based structural diversity of $v$ will then be 0 . From this example, we can see that if the value of $t$ is tuned too high, no social contexts can be identified. But if the value of $t$ is set too low, some strong social contexts with denser structures cannot be captured. Thus, to choose a proper value of $t$ for all vertices in a graph is a challenging task.

To tackle the above issue, we propose a parameter-free scheme for automatically identifying strong social contexts in one's ego-network. We firstly give a novel definition of discriminative core based on the concept of coreness as follows.

Definition 3.4. (Coreness) Given a subgraph $H \subseteq G$, the coreness of $H$ is the minimum degree of vertices in $H$, denoted by $\varphi(H)=\min _{v \in H}\left\{d_{H}(v)\right\}$. The coreness of a vertex $v \in V(G)$ is $\varphi_{G}(v)=\max _{H \subseteq G, v \in V(H)}\{\varphi(H)\}$.

Definition 3.5. (Discriminative Core) Given a graph $G$ and a subgraph $H \subseteq G, H$ is a discriminative core if and only if $H$ is a maximal connected subgraph such that there exists no subgraph $H^{\prime} \subseteq H$ with $\varphi\left(H^{\prime}\right)>\varphi(H)$.

By Def. 3.5, a discriminative core $H$ is a maximal connected component that cannot be further decomposed into smaller subgraphs with a higher coreness. It indicates that a discriminative core is the densest and most important component of a social context, which can be used as a distinct element to represent a social context. In addition, the coreness of a discriminative core reflects 
the strength of its representative social context. For example, $H_{4}$ is a discriminative core with $\varphi\left(H_{4}\right)=3$. And $H_{2}$ is another discriminative core with $\varphi\left(H_{2}\right)=2$. According to the core-based structural diversity, they cannot be identified as distinct social contexts simultaneously using the same value of parameter $t$. But by our discriminative core definition, they will be treated as distinct social contexts automatically without loosing the information of their strength.

For an ego-network $G_{N(v)}$, the whole network may consist of multiple discriminative cores with various corenesses, which can be depicted as a coreness distribution of discriminative cores. Moreover, to rank the structural diversity of two vertices, it is difficult to directly compare the coreness distributions of two ego-networks. Because it is not easy to measure both the number of social contexts and the strength of social contexts simultaneously.

Making use of the idea of $h$-index criteria, we define the diversity vector and diversity score as follows.

Definition 3.6. (Diversity Vector and Diversity Score) Given a graph $G$ and a vertex $v$, the diversity vector of $v$ is the coreness distribution of discriminative cores in $G_{N(v)}$, denoted by $C(v)=\left[c_{v}(1), \ldots, c_{v}(n)\right]$, where $c_{v}(r)=\mid\left\{H: \varphi(H)=r\right.$ and $H$ is a discriminative core in $\left.G_{N(v)}\right\} \mid$. The $h$-index based structural diversity score of $v$, denoted by $h(v)$, is defined as $h(v)=\max \{r$ : $\left.\sum_{r}^{n} c_{v}(r) \geq r\right\}$. For short, diversity score is called.

Example 3.7. Consider the ego-network of $v$ shown in Fig. 1, subgraph $H_{1}$ is not a 2-core discriminative component since it can be further decomposed into two 3-cores $\mathrm{H}_{4}$ and $\mathrm{H}_{5}$. There is no discriminative core with the coreness of 1 , so $c_{v}(1)=0$. And $c_{v}(2)=2$ since it has two discriminative cores $\mathrm{H}_{2}$ and $\mathrm{H}_{3}$ with the coreness of 2 . Similarly, $c_{v}(3)=2$ because $H_{4}, H_{5}$ are two discriminative cores with the coreness of 3 . There exists no discriminative cores with coreness greater than 3 . Thus, the diversity vector of $v$ is $C(v)=[0,2,2,0, \ldots, 0]$. And the diversity score is $h(v)=2$ by definition.

In this paper, we study the problem of $h$-index based structural diversity search in a graph. The problem formulation is defined as follows.

Problem Formulation. Given a graph $G$ and an integer $k$, the goal of $h$-index based structural diversity search problem is to find an optimal answer $S^{*}$ consisted of $k$ vertices with the highest $h$-index based structural diversity scores, i.e.,

$$
S^{*}=\underset{S \subseteq V,|S|=k}{\arg \max }\left\{\min _{v \in S} h(v)\right\} .
$$

\section{BASELINE ALGORITHM}

In this section, we introduce a baseline approach for h-index based structural diversity search over graph $G$. The high-level idea is to compute the diversity score for each vertex in graph $G$ one by one. After obtaining the scores of all vertices, it sorts vertices in decreasing order of their scores and returns the first $k$ vertices with the highest structural diversity scores. This method computes the top- $k$ result from scratch, which is intuitive and straightforward to obtain answers.

In the following, we first introduce an existing algorithm of core decomposition [2]. Then, we present an important and useful procedure to compute h-index based structural diversity score $h(v)$ for a given vertex $v$.

\subsection{Core Decomposition}

The core decomposition of graph $G$ computes the coreness of all vertices $v \in V$. Algorithm 1 outlines the algorithm of core decomposition [2]. The algorithm starts with an integer $t=1$, and iteratively removes the nodes with degree less than $t$ and their incident edges. The number of $t-1$ 


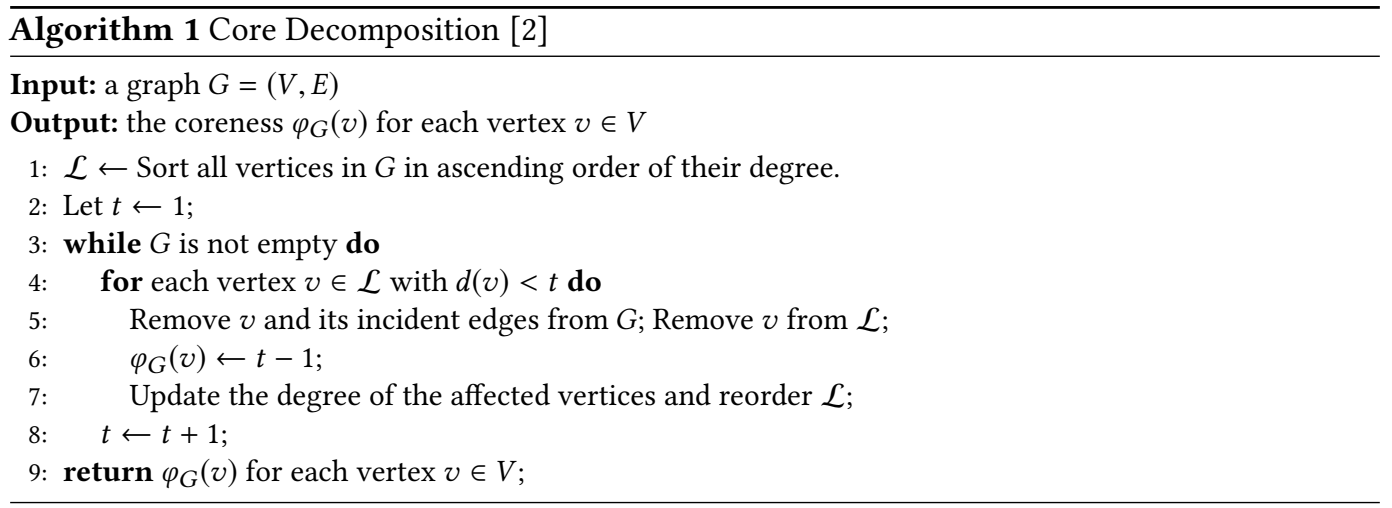

is assigned to be the coreness of the removed vertices. Then, the degree of affected vertices needs to be updated, since the removal of a vertex decreases the degree of its neighbors in the remaining graph. The number $t$ is increased by one after each iteration, until all vertices and edges are deleted from the input graph.

\subsection{Computing $h(v)$}

The computation of $h(v)$ includes three major steps. First, we extract from graph $G$ and obtain an ego-network $G_{N(v)}$ for vertex $v$, which is the induced subgraph of $G$ by the set of $v$ 's neighbors $N(v)$. Next, we decompose the entire ego-network $G_{N(v)}$ into several discriminative cores, and count their corenesses to derive structural diversity vector $C(v)$. The detailed procedure is outlined in Algorithm 2. Finally, based on the diversity vector of $C(v)$, we compute the diversity score $h(v)$ by the Def. 3.6 using Algorithm 3.

Discriminative Core Decomposition. Algorithm 2 outlines the detailed steps for discriminative core decomposition and diversity vector computation. For an ego-network $G_{N(v)}$ of vertex $v$, we firstly apply the core decomposition algorithm on it to calculate the coreness of each vertex (line 1). Then, we sort all vertices in $G_{N(v)}$ in ascending order of their coreness (line 3). For each integer $t$ from 1 to the maximum coreness of the vertices in $G_{N(v)}$, we identify and count the number of discriminative cores with the coreness of $t$ by using a breadth first search approach (lines 5-19). By definition, a discriminative core with the coreness of $t$ will be only formed by the vertices with the coreness of exactly $t$. Thus, in each iteration, we traverse vertices with the same coreness of $t$ to search all the discriminative cores $H$ s with $\varphi(H)=t$ (lines 7-19 and lines 14-15). Edges connecting the current visited vertex $x$ to the vertices with coreness greater than $t$ indicate that the current found component can not be counted as a discriminative core and $x$ does not belong to any discriminative cores in $G_{N(v)}$ (lines 16-17). Then the $t$-th element $c_{v}(t)$ of the diversity vector $C(v)$ can be computed (lines 18-19). Finally, the diversity vector $C(v)$ of $v$ will be returned.

$H$-index Score Computation. The details of computing the $h$-index based structural diversity score are shown in Algorithm 3. After figuring out the diversity vector $C(v)$ (lines 1-2), the diversity score $h(v)$ can then be calculated by Def. 3.6 (lines 3-6). We firstly initialize $h(v)$ as 0 (line 3). Then, for each element $c_{v}(t)$ in the reverse order of the diversity vector $C(v)$, we keep accumulating it to $h(v)$ until the first $t$ appears such that $h(v) \geq t$ (line 4-6). Such $t$ is the diversity score $h(v)$ of $v$.

Equipped with Algorithm 3, we are able to compute the $h$-index based structural diversity for all the vertices in $G$. By sorting the diversity scores, we can obtain the top- $k$ results for a given $k$. 

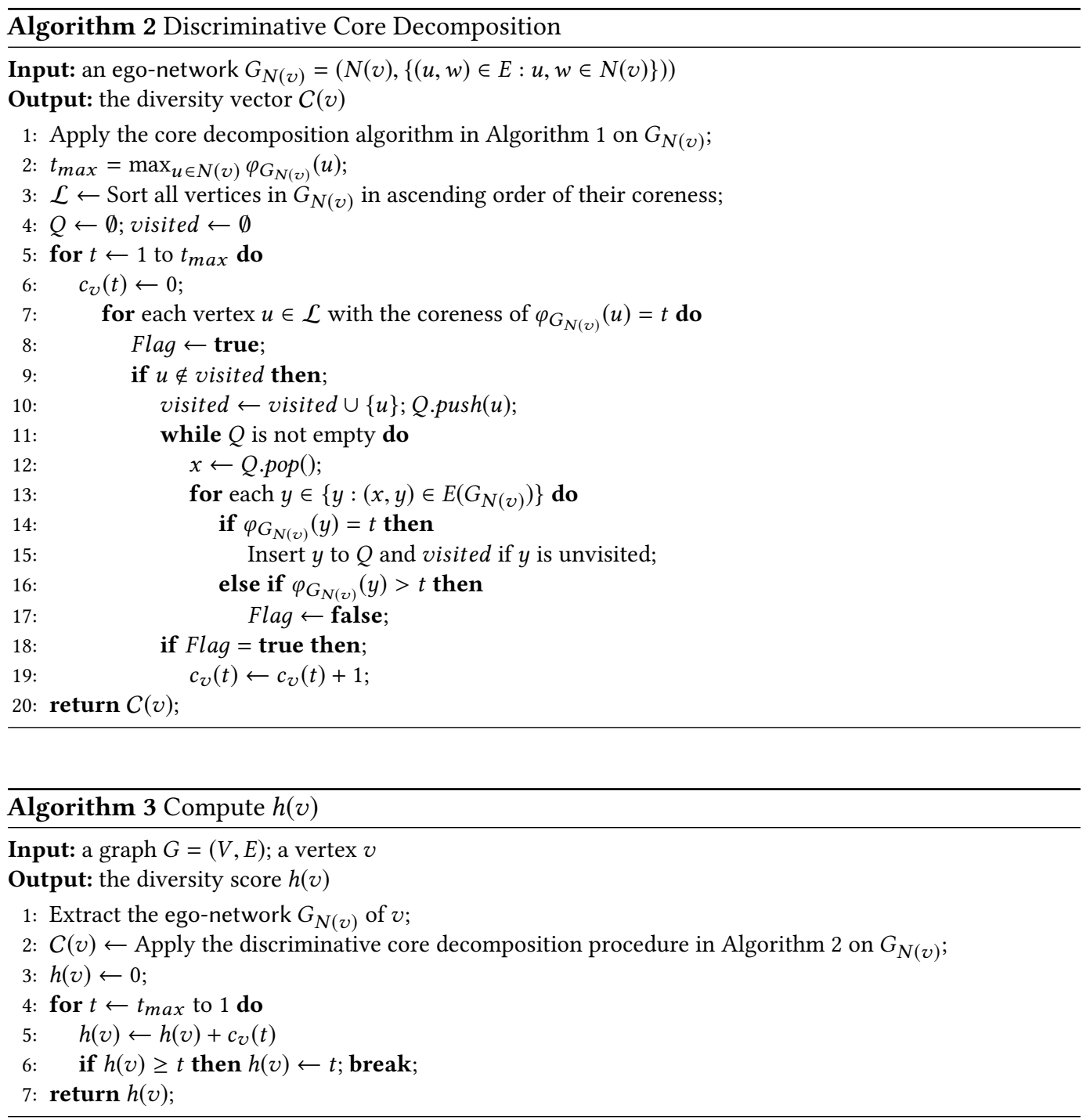

\section{EFFICIENT TOP- $k$ SEARCH ALGORITHM}

The drawback of baseline method presented in the previous section is obviously inefficient and can be improved. Firstly, both the ego-network extraction and discriminative core decomposition are costly in computation. Secondly, it iteratively computes the $h$-index based structural diversity scores for all vertices on the entire graph $G$, which is expensive. Thirdly, some vertices appear to be obviously unqualified for the top- $k$ result. And the score computations of them are reluctant and should be avoided.

In this section, we develop an efficient top- $k$ search framework by exploiting useful pruning techniques to reduce the search space, leading to a small number of candidate vertices for score computations. Specifically, we design an upper bound $\widehat{h}(v)$ for diversity score $h(v)$, based on the analysis of the core structure. 


\subsection{An Upper Bound of $h(v)$}

We starts with a structural property of $t$-core.

Lemma 5.1. Given a vertex $v$ and any vertex $u \in N(v)$, if $u$ has $\varphi_{G_{N(v)}}(u)=r$ in ego-network $G_{N(v)}$, then $u$ has the coreness $\varphi_{G}(u) \geq r+1$ in graph $G$.

Proof. We omit the proof for brevity. The detailed proof can be referred to [12].

Example 5.2. Consider vertex $x_{1}$ in Fig. $1, x_{1}$ has coreness $\varphi_{G}\left(x_{1}\right)=4$. However, in the egonetwork $G_{N(v)}, \varphi_{G_{N(v)}}\left(x_{1}\right)=3$. Here $\varphi_{G}\left(x_{1}\right) \geq \varphi_{G_{N(v)}}\left(x_{1}\right)+1$ holds.

For a vertex $v$ and some vertices $u \in N(v)$, the global coreness $\varphi_{G}(u)$ is sometimes much larger than the coreness of $u$ in the ego-network of $v$, i.e. $\varphi_{G}(u)>>\varphi_{G_{N(v)}}(u)$. The following lemma gives another upper bound for estimating the coreness $\varphi_{G_{N(v)}}(u)$, w.r.t. vertices $v$ and $u \in N(v)$.

Lemma 5.3. Given a vertex $v$ and its coreness $\varphi_{G}(v), \forall u \in N(v), \varphi_{G_{N(v)}}(u)<\varphi_{G}(v)$.

Proof. We prove this by contradiction. For any $u \in N(v)$, we assume $\varphi_{G}(v)=r$ and $\varphi_{G_{N(v)}}(u) \geq$ $\varphi_{G}(v)$, which is $\varphi_{G_{N(v)}}(u) \geq r$. By the definition of coreness, there exists a subgraph $H \subseteq G_{N(v)}$ with coreness $\varphi(H) \geq r$ indicating that $\forall v^{*} \in V(H), d_{H}\left(v^{*}\right) \geq r$. We add the vertex $v$ and its incident edges to $H$ to generate a new subgraph $H^{\prime} \subseteq G$, where $V\left(H^{\prime}\right)=V(H) \cup\{v\}$ and $E\left(H^{\prime}\right)=E(H) \cup\{(v, u): u \in V(H)\}$. It's easy to verify that for all $v^{*}$ in $H^{\prime}$, we have $d_{H^{\prime}}\left(v^{*}\right) \geq r+1$. Since $v$ is also contained in $H^{\prime}$, by definition, $\varphi_{G}(v) \geq r+1$, which contradicts to the condition $\varphi_{G}(v)=r$.

Combining Lemma 5.1 and Lemma 5.3, we have the following corollary.

Corollary 5.4. Given a vertex $v$ in graph $G$, for any vertex $u \in N(v), \widehat{\varphi}_{G_{N(v)}}(u)=\min \left\{\varphi_{G}(v), \varphi_{G}(u)-\right.$ 1) and $\widehat{\varphi}_{G_{N(v)}}(u) \geq \varphi_{G_{N(v)}}(u)$ hold.

Based on Corollary 5.4, we derive an upper bound $\widehat{h}(v)$ for the $h$-index based structural diversity score $h(v)$ as follows.

LEMMA 5.5. Given a vertex $v$ and its ego-network $G_{N(v)}$, we have an upper bound of diversity score $h(v)$, denoted by

$$
\widehat{h}(v)=\max _{x \in \mathbb{Z}_{+}}\left\{x:\left|\left\{u \in N(v): \widehat{\varphi}_{G_{N(v)}}(u) \geq x\right\}\right| \geq x \cdot(x+1)\right\} .
$$

Proof. Assume that $h(v)=x^{*}$, we prove $\widehat{h}(v) \geq x^{*}$. By $h(v)=x^{*}$, it indicates that there exists $x^{*}$ discriminative cores $g$ with $\varphi(g) \geq x^{*}$ in the ego-network $G_{N(v)}$. For $\varphi(g) \geq x^{*}$, discriminative core $g$ has at least $x^{*}+1$ nodes $u$ with $\varphi_{G_{N(v)}}(u) \geq x^{*}$. Thus, the whole ego-network $G_{N(v)}$ has at least $x^{*} \cdot\left(x^{*}+1\right)$ nodes $u$ with $\varphi_{G_{N(v)}}(u) \geq x^{*}$, i.e., $h(v)=x^{*} \leq \max _{x \in \mathbb{Z}_{+}}\left\{x: \mid\left\{u \in N(v): \varphi_{G_{N(v)}}(u) \geq\right.\right.$ $x\} \mid \geq x \cdot(x+1)\}$. By Corollary 5.4, $\widehat{\varphi}_{G_{N(v)}}(u) \geq \varphi_{G_{N(v)}}(u)$, hence we have $\widehat{h}(v) \geq x^{*}=h(v)$.

According to Lemma 5.5, once applying the core decomposition algorithm on graph $G$, we can directly compute the upper bounds $\widehat{h}(v)$ for all vertices $v$.

\subsection{Top-K Structural Diversity Search Framework}

Equipped with the upper bound $\widehat{h}(v)$, we develop an efficient top- $k$ search framework for safely pruning the search space and avoiding the unnecessary computation of $h(v)$. The efficient top-k structural diversity search framework is presented in Algorithm 4.

Algorithm 4 starts with the initialization of the upper bound of each vertex $v$ (lines 1-2). Then, it sorts all vertices in descending order according to their upper bounds (line 3). It maintains a list 


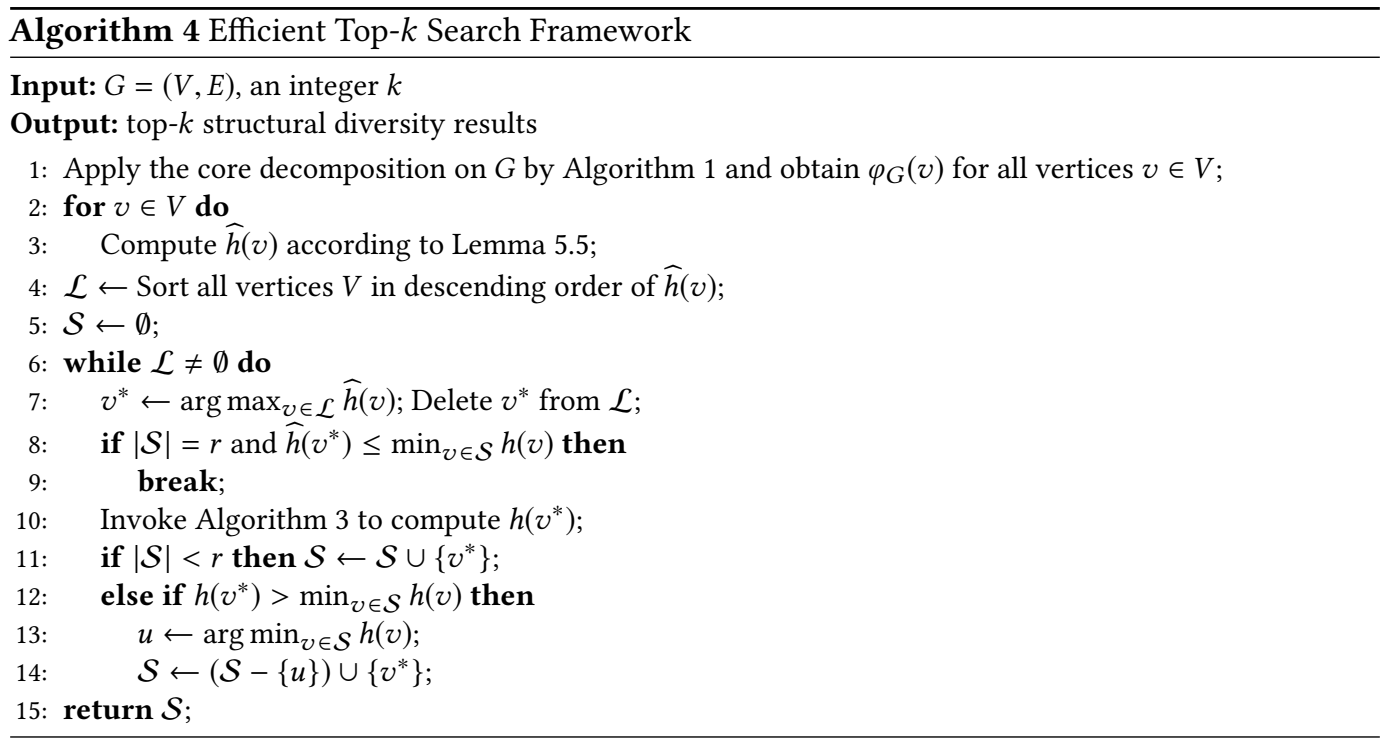

$\mathcal{S}$ to store the top- $k$ result (line 4 ). In each iteration, the algorithm pops out a vertex $v^{*}$ from the vertex list $\mathcal{L}$ with the largest upper bound $\widehat{h}\left(v^{*}\right)$ (line 6$)$. Next, it checks the early stop condition: if the answer set $\mathcal{S}$ has $k$ results and the minimum score in $\mathcal{S}$ is no less than the current upper bound, i.e. $\widehat{h}\left(v^{*}\right) \leq \min _{v \in \mathcal{S}} h(v)$, the current vertex $v^{*}$ is safely pruned and the searching process is terminated (lines 8-9). Otherwise, the procedure of structural diversity score computation is invoked and check if $v^{*}$ can be added into the result set (lines 10-14). Finally, the top- $k$ results stored in $\mathcal{S}$ are returned.

\subsection{Complexity Analysis}

In this section, we analyze the time and space complexity of Algorithm 4.

Lemma 5.6. Algorithm 3 computes $h(v)$ for each vertex $v$ in $O\left(\sum_{u \in N(v)} \min \{d(u), d(v)\}\right)$ time and $O(m)$ space.

Proof. Extracting $G_{N(v)}$ of $v$ takes $O\left(\sum_{u \in N(v)} \min \{d(u), d(v)\}\right)$, since all triangles $\triangle_{v u w}$ should be listed to enumerate each edge $(u, w) \in E\left(G_{N(v)}\right)$. According to [2], the core decomposition performed in $G_{N(v)}$ takes $O\left(\left|E\left(G_{N(v)}\right)\right|+d(v)\right)$ time. The sorting of the vertices can be finished in $O(d(v))$ time using bin sort. And the breadth first search process for identifying the discriminative cores needs $O\left(\left|E\left(G_{N(v)}\right)\right|\right)$ time. In addition, the computing of the $h$-index based structural diversity score $h(v)$ runs in $O\left(\delta\left(G_{N(v)}\right)\right)$ time, where $\delta\left(G_{N(v)}\right)=\max _{u \in N(v)} \varphi_{G_{N(v)}}(u)$ is the degeneracy of $G_{N(v)}$. And $\delta\left(G_{N(v)}\right)$ is bounded by the degree of $v$, which is $O\left(\delta\left(G_{N(v)}\right) \subseteq d(v)\right.$. Overall, the time complexity of Algorithm 3 is $O\left(\sum_{u \in N(v)} \min \{d(u), d(v)\}\right)$.

We continue to analyze the space complexity of Algorithm 3. The storage of the ego-network of $v$ takes $O(n+m)$ space since $G_{N(v)} \subseteq G$. And both the sorted list of vertices (line 4$)$ and the structural diversity vector of $v$ takes $O(n)$ space. Thus, the space complexity of Algorithm 3 is $O(n+m) \subseteq O(m)$ due to our graph connectivity assumption.

Theorem 5.7. Algorithm 4 computes the top-k results in $O(\rho m)$ time and $O(m)$ space, where $\rho$ is the arboricity of $G$ and $\rho \leq \min \left\{d_{\max }, \sqrt{m}\right\}$ [7]. 
Table 1. Network Statistics

\begin{tabular}{|c|c|c|c|}
\hline Name & $|V|$ & $|E|$ & $d_{\max }$ \\
\hline \hline Gowalla & 196,591 & 950,327 & 14,730 \\
\hline Youtube & $1,134,890$ & $2,987,624$ & 28,754 \\
\hline LiveJournal & $3,997,962$ & $34,681,189$ & 14,815 \\
\hline Orkut & $3,072,441$ & $117,185,083$ & 33,313 \\
\hline
\end{tabular}

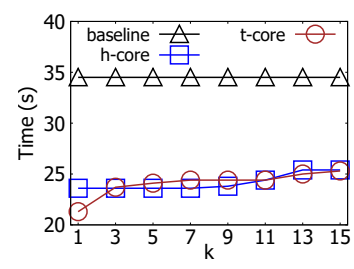

(a) Gowalla

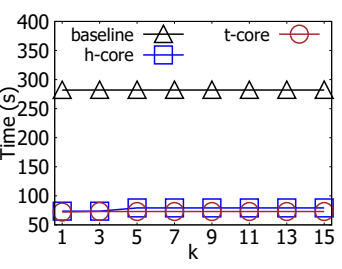

(b) Youtube

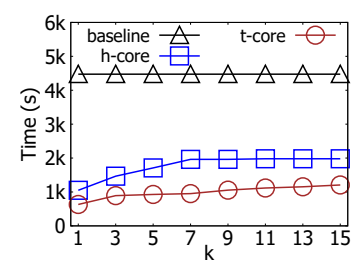

(c) LiveJournal

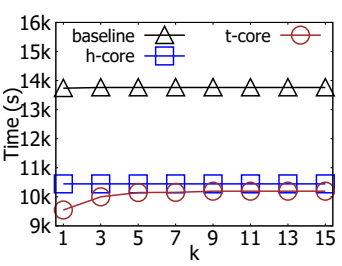

(d) Orkut

Fig. 2. Comparsion of baseline, h-core and t-core in terms of running time (in seconds).

Proof. Firstly, the core decomposition algorithm performed on $G$ takes $O(m)$ time and $O(n+m)$ space. Secondly, the computation of upper bound $\widehat{h}(v)$ for all $v$ 's takes $O(m)$ time and $O(n)$ space. In the worst case, Algorithm 4 needs to compute $h(v)$ for every vertex $v$. This takes $O\left(\sum_{v \in V}\left\{\sum_{u \in N(v)} \min \{d(u), d(v\right.\right.$ time in total by Lemma 5.6. According to [7], we have

$$
O\left(\sum_{v \in V}\left\{\sum_{u \in N(v)} \min \{d(u), d(v)\}\right\}\right) \subseteq O\left(\sum_{(u, v) \in E} \min \{d(u), d(v)\}\right) \subseteq O(\rho m) .
$$

Here $\rho$ is the arboricity of graph $G$, which is defined as the minimum number of disjoint spanning forests that cover all the edges in $G$. In addition, the top- $k$ results can be maintained in a list in $O(n)$ time and $O(n)$ space using bin sort. Overall, Algorithm 4 runs in $O(\rho m)$ time and $O(m)$ space.

\section{EXPERIMENTS}

We conduct extensive experiments on real-world datasets to evaluate the effectiveness and efficiency of our proposed $h$-index based structural diversity model and algorithms.

Datasets: We run our experiments on four real-world datasets downloaded on the SNAP website [18]. All datasets are treated as undirected graphs. The statistics of the networks are listed in Table 1 . We report the node size $|V|$, edge size $|E|$ and the maximum degree $d_{\text {max }}$ of each network.

Compared Methods: We evaluate all compared methods in terms of efficiency, effectiveness and also sensitivity to parameter setting. Specifically, we show three compared algorithms as follows.

- baseline: is the baseline method proposed in Section 4.

- h-core: is an improved top- $k$ search algorithm for computing the top- $k$ vertices with highest $h$-index based structural diversity in Algorithm 4.

- $\mathrm{t}$-core: is to compute the top- $k$ vertices with highest $t$-core based structural diversity [12]. Here, $t$ is a parameter of coreness threshold.

Note that in the sensitivity evaluation, we test the state-of-the-art competitor t-core and compare the top- $k$ results for different parameter $t$. Our $h$-index based structural diversity model has no input parameter, which is consistent on the top- $k$ results.

\subsection{Efficiency Evaluation}

In this experiment, we compare the efficiency of baseline, $\mathrm{h}$-core and $\mathrm{t}$-core on four real-world datasets. For the t-core method, we fix parameter $t=2$. We compare the running time and search space (i.e., the number of vertices whose structural diversity scores are computed in the search 


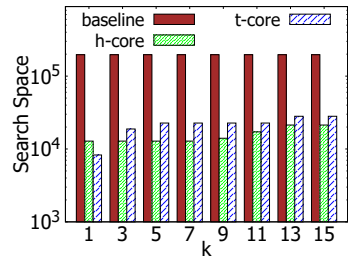

(a) Gowalla

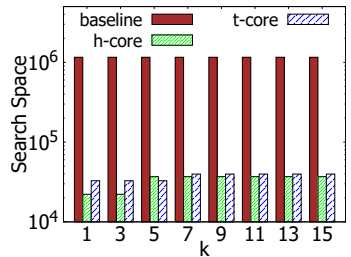

(b) Youtube

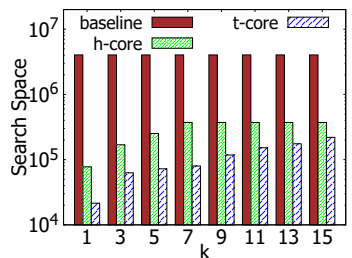

(c) LiveJournal

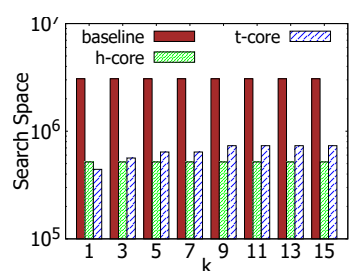

(d) Orkut

Fig. 3. Comparsion of baseline, h-core and t-core in terms of search space.

process). Fig. 2 shows the running time results of three methods varied by $k$. It clearly shows that top- $k$ search algorithm $\mathrm{h}$-core runs much faster than baseline on all the reported datasets. Specifically, in Fig. 2(c), h-core is 5 times faster than baseline on Youtube in term of running time. Moreover, Fig. 3 further shows the search space of three methods varied on all datasets. We can observe that leveraging on the upper bound $\widehat{h}(v)$, a large number of disqualified vertices is pruned during the search process by h-core. The search space significantly shrinks into less than $\frac{1}{10}$ of vertex size in graphs. It verifies the tightness of our upper bound and the superiority of $\mathrm{h}$-core against baseline in efficiency. According to Fig. 2 and Fig. 3, our h-core is very comparative to the state-of-art method t-core in terms of running time and search space.

\subsection{Sensitivity Evaluation}

This experiment evaluates the sensitivity of $\mathrm{t}$-core model. Given two different values of $t$, $\mathrm{t}$-core model may generate two different lists of top- $k$ ranking results. We use the Kendall rank tau distance to counts the number of pairwise disagreements between two top- $k$ lists. The larger the distance, the more dissimilar the two lists, and also more sensitive the $t$-core model. We adopt the Kendall distance with penalty, denoted by,

$$
K^{(p)}\left(\tau_{1}, \tau_{2}\right)=\sum_{\{i, j\} \in \mathcal{P}} \bar{K}_{i, j}^{(p)}\left(\tau_{1}, \tau_{2}\right)
$$

where $\mathcal{P}$ is the set of all unordered pairs of distinct elements in two top- $k$ list $\tau_{1}, \tau_{2}$ and $p$ is the penalty parameter. In our setting, we set $p=1$ and normalize the Kendall distance by the number of permutation $|\mathcal{P}|$. The values of normalized Kendall distance range from 0 to 1 .

We test the sensitivity of $\mathrm{t}$-core model by varying parameter $t$ in $\{2,4,6,8,10\}$. We compute the Kendall distance of two top-100 lists by t-core model with two different $t$. The results of sensitivity heat matrix on four datasets are shown in Fig. 4. The darker colors reveal larger Kendall distances between two top- $k$ lists and also more sensitive of $\mathrm{t}$-core models on this pair of parameters $t$. Overall, sensitivity heat matrices are depicted in dark for most parameter settings on all datasets. This reflects that the top- $k$ results computed by $\mathrm{t}$-core are very sensitive to the setting of parameter $t$, which has a bad robustness. It strongly indicates the necessity and importance of our parameter-free structural diversity model.

\subsection{Effectiveness Evaluation}

In this experiment, we evaluate the effectiveness of our proposed $h$-index based structural diversity. We compare our method h-core with state-of-the-art t-core [12] in the task of social contagion. Specifically, we adopt the independent cascade model to simulate the influence propagation process in graphs [10]. Influential probability of each edge is set to 0.01 . Then, we select 50 vertices as activated seeds by an influence maximization algorithm [24]. We perform 1000 times of Monte Carlos sampling for propagation. For comparison, we count the number of activated vertices in 


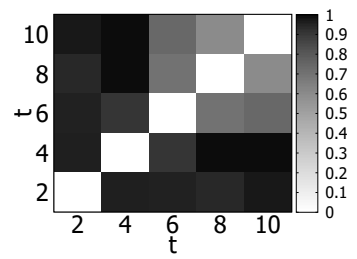

(a) Gowalla

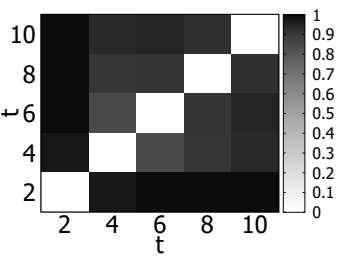

(b) Youtube

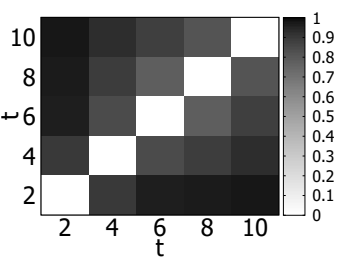

(c) LiveJournal

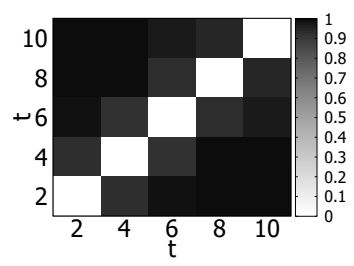

(d) Orkut

Fig. 4. Sensitivity heat matrices of t-core model on all datasets. Each matrix element represents the Kendall's Tau distance between two top-100 ranking lists by t-core model with different $t$.

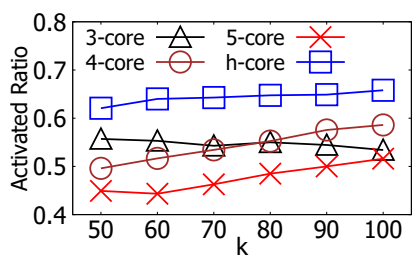

(a) Average Activated Rate

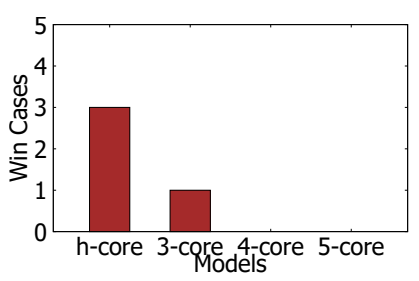

(b) Win Cases Distribution

Fig. 5. Comparison of t-core and h-core in terms of the average activated ratio and win cases on four datasets.

the top- $k$ results by $\mathrm{t}$-core and $\mathrm{h}$-core methods. The method that achieves the largest number of activated vertices is regarded as the winner.

First, we report the average activated rate by h-core and t-core method on all four datasets in Fig. 5(a). Let $D=\{$ “Gowalla","Youtube", "LiveJournal","Orkut” $\}$. Given a dataset $d \in D$, the activated rate is defined as $f_{k}(d)=\frac{A_{c t N u m_{k}}}{k}$, where ActNum $_{k}$ is the number of activated vertices in the top- $k$ result. The average activated rate is defined as ActRate $k_{k}=\frac{\sum_{d \in D} f_{k}(d)}{|D|}$. Fig. 5(a) shows that our method h-core achieves the highest activated rates, which significantly outperforms t-core method for all different $t$. It indicates that the top- $k$ results found by h-core tend to have higher probability to be affected in social contagion.

In addition, we also report the win cases of h-core and t-core with different parameter $t$ on all dataset. We vary $t=\{2,3,4\}$ and set $k=100$ for all methods. The winner of a dataset is the method that achieves the highest number of activated vertices in this dataset. Fig. $5(\mathrm{~b})$ shows the win cases of t-core and h-core. As we can see, h-core wins on three datasets, which achieves the best performance. It further shows the superiority of our $h$-index structural diversity model. Besides, 3-core wins once, 4-core and 5-core win none, indicating that $\mathrm{t}$-core performs sensitively to parameter $t$.

\section{CONCLUSION}

In this paper, we propose a parameter-free structural diversity model based on $h$-index and study the top- $k$ structural diversity search problem. To solve the top- $k$ structural diversity search problem, an upper bound for the diversity score and a top- $k$ search framework for efficiently reducing the search space are proposed. Extensive experiments on real-wold datasets verify the efficiency of our pruning techniques and the effectiveness of our proposed $h$-index based structural diversity model.

\section{ACKNOWLEDGMENTS}

This work is supported by the NSFC Nos. 61702435, 61972291, RGC Nos. 12200917, 12200817, CRF C6030-18GF, and the National Science Foundation of Hubei Province No. 2018CFB519. 


\section{REFERENCES}

[1] Sabeur Aridhi, Martin Brugnara, Alberto Montresor, and Yannis Velegrakis. 2016. Distributed k-core decomposition and maintenance in large dynamic graphs. In DEBS. ACM, 161-168.

[2] V. Batagelj and M. Zaversnik. 2003. An O (m) algorithm for cores decomposition of networks. arXiv preprint cs/0310049 (2003).

[3] Francesco Bonchi, Francesco Gullo, Andreas Kaltenbrunner, and Yana Volkovich. 2014. Core decomposition of uncertain graphs. In KDD. ACM, 1316-1325.

[4] Lijun Chang, Chen Zhang, Xuemin Lin, and Lu Qin. 2017. Scalable Top-K Structural Diversity Search. In ICDE. 95-98.

[5] Huanyu Cheng, Ming Zhong, Jian Wang, and Tieyun Qian. 2019. Keyword Search Based Mashup Construction with Guaranteed Diversity. In DEXA. 423-433.

[6] James Cheng, Yiping Ke, Shumo Chu, and M. Tamer Özsu. 2011. Efficient core decomposition in massive networks. In ICDE. 51-62.

[7] Norishige Chiba and Takao Nishizeki. 1985. Arboricity and Subgraph Listing Algorithms. SIAM J. Comput. 14, 1 (1985), 210-223.

[8] Fei Ding and Yi Zhuang. 2018. Ego-network probabilistic graphical model for discovering on-line communities. Appl. Intell. 48, 9 (2018), 3038-3052.

[9] Edoardo Galimberti, Francesco Bonchi, and Francesco Gullo. 2017. Core Decomposition and Densest Subgraph in Multilayer Networks. In CIKM. ACM, 1807-1816.

[10] Amit Goyal, Wei Lu, and Laks V. S. Lakshmanan. 2011. CELF++: optimizing the greedy algorithm for influence maximization in social networks. In WWW. 47-48.

[11] Jorge E Hirsch. 2005. An index to quantify an individual's scientific research output. Proceedings of the National academy of Sciences 102, 46 (2005), 16569-16572.

[12] Xin Huang, Hong Cheng, Rong-Hua Li, Lu Qin, and Jeffrey Xu Yu. 2015. Top-K structural diversity search in large networks. VLDB f. 24, 3 (2015), 319-343.

[13] Xin Huang, Hong Cheng, Rong-Hua Li, Lu Qin, and Jeffrey Xu Yu. 2013. Top-K Structural Diversity Search in Large Networks. PVLDB 6, 13 (2013), 1618-1629.

[14] Xin Huang, Laks VS Lakshmanan, and Jianliang Xu. 2019. Community search over big graphs. Morgan \& Claypool Publishers.

[15] R Robert Huckfeldt and John Sprague. 1995. Citizens, politics and social communication: Information and influence in an election campaign. Cambridge University Press.

[16] Paul Jakma, Marcin Orczyk, Colin S. Perkins, and Marwan Fayed. 2012. Distributed k-core decomposition of dynamic graphs. In StudentWorkshop@CoNEXT. ACM, 39-40.

[17] David Kempe, Jon M. Kleinberg, and Éva Tardos. 2003. Maximizing the spread of influence through a social network. In KDD. 137-146.

[18] Jure Leskovec and Andrej Krevl. 2014. SNAP Datasets: Stanford Large Network Dataset Collection. http://snap.stanford. $\mathrm{edu} /$ data.

[19] Vincent Levorato. 2014. Core Decomposition in Directed Networks: Kernelization and Strong Connectivity. In CompleNet, Vol. 549. 129-140.

[20] Rong-Hua Li, Jeffrey Xu Yu, and Rui Mao. 2014. Efficient Core Maintenance in Large Dynamic Graphs. TKDE 26, 10 (2014), 2453-2465.

[21] Julian Mcauley and Jure Leskovec. 2014. Discovering social circles in ego networks. TKDD 8, 1 (2014), 4.

[22] Alberto Montresor, Francesco De Pellegrini, and Daniele Miorandi. 2013. Distributed k-Core Decomposition. TPDS 24, 2 (2013), 288-300.

[23] Ahmet Erdem Sarıüce, Bugra Gedik, Gabriela Jacques-Silva, Kun-Lung Wu, and Ümit V. Çatalyürek. 2013. Streaming Algorithms for k-core Decomposition. PVLDB 6, 6 (2013), 433-444.

[24] Youze Tang, Yanchen Shi, and Xiaokui Xiao. 2015. Influence Maximization in Near-Linear Time: A Martingale Approach. In SIGMOD Conference. ACM, 1539-1554.

[25] J. Ugander, L. Backstrom, C. Marlow, and J. Kleinberg. 2012. Structural diversity in social contagion. PNAS 109, 16 (2012), 5962-5966.

[26] Dong Wen, Lu Qin, Ying Zhang, Xuemin Lin, and Jeffrey Xu Yu. 2019. I/O Efficient Core Graph Decomposition: Application to Degeneracy Ordering. TKDE 31, 1 (2019), 75-90.

[27] Huanhuan Wu, James Cheng, Yi Lu, Yiping Ke, Yuzhen Huang, Da Yan, and Hejun Wu. 2015. Core decomposition in large temporal graphs. In BigData. 649-658.

[28] Yikai Zhang, Jeffrey Xu Yu, Ying Zhang, and Lu Qin. 2017. A Fast Order-Based Approach for Core Maintenance. In ICDE. 337-348. 